\title{
Development of a High-Throughput Screening Assay Based on the 3-Dimensional Pannus Model for Rheumatoid Arthritis
}

\author{
YVONNE IBOLD, ${ }^{1,2}$ SIMONE FRAUENSCHUH, ${ }^{1}$ CHRISTIAN KAPS,${ }^{3}$ MICHAEL SITTINGER, ${ }^{2}$ \\ JOCHEN RINGE, ${ }^{2}$ and PETER M. GOETZ ${ }^{1}$
}

\begin{abstract}
The 3-dimensional (3-D) pannus model for rheumatoid arthritis (RA) is based on the interactive co-culture of cartilage and synovial fibroblasts (SFs). Besides the investigation of the pathogenesis of RA, it can be used to analyze the active profiles of antirheumatic pharmaceuticals and other bioactive substances under in vitro conditions. For a potential application in the industrial drug-screening process as a transitional step between 2-dimensional (2-D) cell-based assays and in vivo animal studies, the pannus model was developed into an in vitro high-throughput screening (HTS) assay. Using the CyBi ${ }^{\mathrm{TM}}$-Disk workstation for parallel liquid handling, the main cell culture steps of cell seeding and cultivation were automated. Chondrocytes were isolated from articular cartilage and seeded directly into 96-well microplates in high-density pellets to ensure formation of cartilage-specific extracellular matrix (ECM). Cell seeding was performed automatically and manually to compare both processes regarding accuracy, reproducibility, consistency, and handling time. For automated cultivation of the chondrocyte pellet cultures, a sequential program was developed using the CyBio Control software to minimize shear forces and handling time. After 14 days of cultivation, the pannus model was completed by coating the cartilage pellets with a layer of human SFs. The effects due to automation in comparison to manual handling were analyzed by optical analysis of the pellets, histological and immunohistochemical staining, and real-time PCR. Automation of this in vitro model was successfully achieved and resulted in an improved quality of the generated pannus cultures by enhancing the formation of cartilage-specific ECM. In addition, automated cell seeding and media exchange increased the efficiency due to a reduction of labor intensity and handling time. (Journal of Biomolecular Screening 2007:956-965)
\end{abstract}

Key words: 3-D culture, interactive co-culture, HTS assay, automation, pannus model

\section{INTRODUCTION}

$\mathbf{R}$ HEUMATOID ARTHRITIS (RA) is a chronic, systemic autoimmune disease that occurs in about $1 \%$ to $2 \%$ of the world population, which makes it the most common form of inflammatory arthritis. ${ }^{1}$ It is characterized by inflammation of the synovial joints, hyperplastic growth of the synovium, and abnormal immune responses. The synovial hyperplasia leads to formation of the pannus tissue (Fig. 1), which is a unique feature of RA, distinguishing it from other arthritic conditions. The pannus tissue results from infiltration with large numbers of inflammatory cells

${ }^{1}$ Department of Bioprocess Engineering, Institute of Biotechnology, Technical University of Berlin, Germany.

${ }^{2}$ Tissue Engineering Laboratory and Berlin-Brandenburg Center for Regenerative Therapies, Department of Rheumatology and Clinical Immunology, CharitéUniversitätsmedizin Berlin, Germany.

${ }^{3}$ TransTissue Technologies GmbH, Berlin, Germany.

Received Sep 15, 2006, and in revised form Jul 10, 2007. Accepted for publication Jul 16, 2007.

Journal of Biomolecular Screening 12(7); 2007

DOI: $10.1177 / 1087057107307147$ and from an increase in the number of synovial fibroblasts (SFs), which are believed to be key players in the pathogenesis of RA. ${ }^{2}$ The thickened pannus tissue shows an aggressive invasive behavior, ultimately destroying the underlying cartilage and bone. ${ }^{1}$

Because the etiology of RA is still not completely understood and the current medications are able only to alleviate the symptoms or slow down the progression of this disease, RA is a focus for development of new cell-based drug discovery assays. The traditional 2-D cell culture systems offer standardized and easily reproducible conditions for drug testing, but they lack the complex network of cell-cell and cell-matrix interactions of the 3-D in vivo environment. Monolayer cultures lead to morphological changes often accompanied by the loss of tissue-specific gene expression and dedifferentiation. ${ }^{3}$ Chondrocytes, for instance, start to show a fibroblast-like morphology. The collagen synthesis is switched from collagen type II, which is specific for articular cartilage, to collagen type I. ${ }^{4}$ Therefore, the significance of 2-D cell-based screening in predicting the clinical response to new components is limited. ${ }^{5}$ These problems might be avoided by the use of animal models, but this strategy comprises other disadvantages such as the lack of transferability from animal to human organisms, high cost, and ethical issues. 


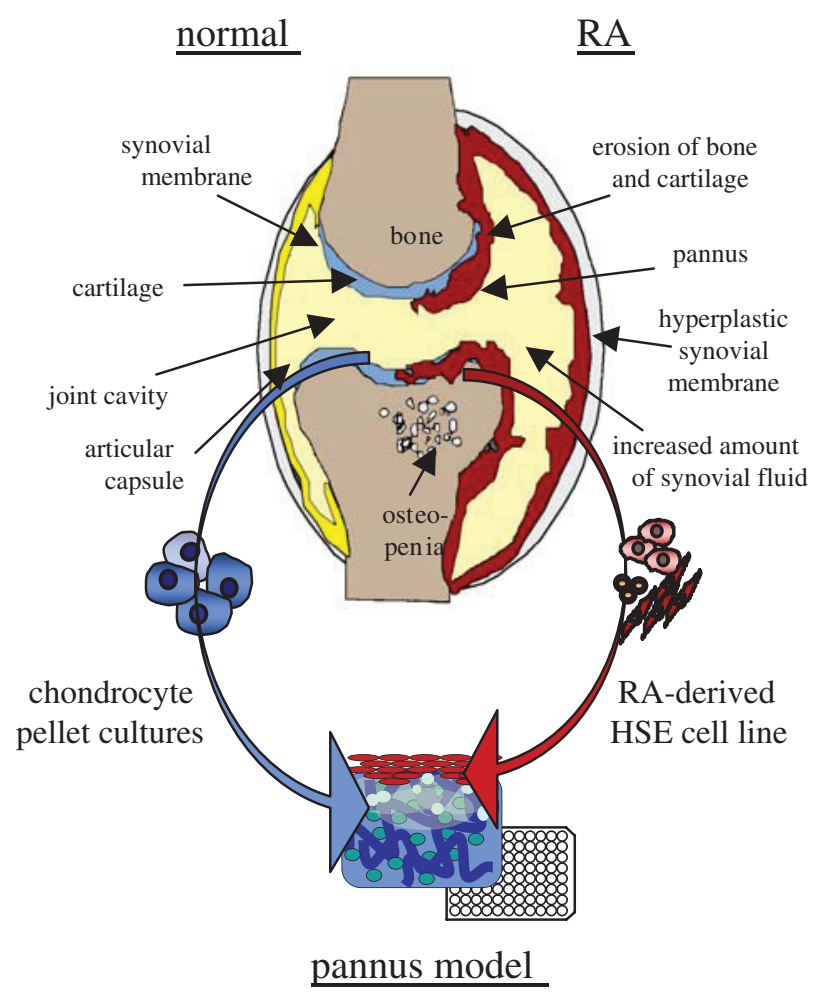

FIG. 1. The 3-dimensional (3-D) in vitro pannus model. The most characteristic feature of rheumatoid arthritis (RA) is the formation of an invasive pannus tissue in the synovial joints. One of the key players in pannus formation is the RA synovial fibroblasts (SFs), which show an aggressive, invasive behavior. During pathogenesis, the pannus tissue ultimately leads to erosion of the underlying cartilage and bone. For simulation of RA in the 3-D pannus model, chondrocytes are harvested from the femoral condyle of healthy donors and cultivated as a high-density pellet culture for 14 days in 96-well microplates to ensure formation of cartilage-specific extracellular matrix. Afterward, the cartilage pellets are coated with a layer of human RA SFs. As the synovial component the rheumatoid HSE cell line is available. The co-culture is maintained for another 7 or 14 days to allow interactions between the different cell types and then can be used for medical drug screening.

To avoid the limitations of 2-D monolayer cultures and animal models, the interactive pannus model was established as a 3-D tissue model for investigation of the pathogenesis of RA. ${ }^{6}$ It was developed as an in vitro test system for the effect of antirheumatic drugs and other bioactive substances and consists of a highdensity pellet culture of chondrocytes and cartilage-specific extracellular matrix (ECM) that is coated with a layer of human SFs (Fig. 1). It has been reported that initial plating of chondrocytes in high-density cultures resulted in the formation of a functional cartilage-like tissue that showed similar responses to interleukin$1 \beta$ as native cartilage explants. ${ }^{7}$ Moreover, cultures that are formed by pelleting chondrocytes followed by cultivation in centrifuge tubes show similar features regarding cellular distribution, matrix composition and density, and tissue ultrastructure compared with native cartilage. ${ }^{8}$ Pellet cultures have been widely used to study epiphyseal and growth plate chondrocyte differentiation and cartilage hypertrophy. ${ }^{9-11}$ The detailed growth characterization of high-density cartilage pellets in microplates and their use in the interactive pannus model was previously reported by the authors. ${ }^{12}$ Pellet-cultured chondrocytes form cartilage-specific ECM by expression of proteoglycans and collagen type II. ${ }^{12,13}$ This 3-D ECM reflects the specific cellular environment of the chondrocytes, allowing functional interactions between the cells and their ECM. For co-cultivation, 2 SV40T antigen-transformed human SF cell lines are available: 1) The hepatic sinusoidal endothelium (HSE) cell line was derived from an RA patient and shows an aggressive invasive behavior in vitro, whereas 2) the K4IM cell line was established from a healthy donor and represents the noninvasive phenotype. ${ }^{14}$ Co-cultivation using these 2 cell lines reflects important aspects of arthritic and healthy conditions and allows investigating the effect of pharmaceuticals and other bioactive substances in drug screening. Recently, HSE and K4IM SFs have been shown to be well suited for testing of frequently used antirheumatic drugs such as methotrexate and prednisolone. Genomewide gene expression profiling of SFs demonstrated that HSE cells express RA-related genes and that this expression profile is reverted or at least directed to normal by treatment with antirheumatic drugs. ${ }^{15}$ For a potential application of the pannus model in the drug-screening process as a transitional step between 2-D cell-based assays and complex animal testing, the main steps for generation of the pannus cultures such as cell seeding and cultivation were automated. For this purpose, the liquid-handling workstation $\mathrm{CyBi}^{\mathrm{TM}}$-Disk allowing parallel handling in a 96-well format was used. Cell seeding and cultivation were performed automatically and manually to investigate the effect of the automation process on the pannus cultures. The automated cell-seeding process was analyzed for accuracy, reproducibility, consistency, and saving in handling time. The quality of the cultivated cartilage pellets after automated and manual cultivation was studied by optical analysis, histology, immunohistochemistry, and real-time PCR.

\section{MATERIALS AND METHODS}

\section{Isolation of chondrocytes}

Articular cartilage was harvested from the medial and lateral condyle of the knee joints of femur bones of 6- to 10-month-old porcine donors. The cartilage slices were diced and incubated in a stirred spinner flask containing RPMI 1640 medium (Biochrom, Berlin, Germany) supplemented with $10 \%$ fetal bovine serum (FBS; Biochrom), $100 \mathrm{U} / \mathrm{ml}$ penicillin and $100 \mu \mathrm{g} / \mathrm{ml}$ streptomycin (Biochrom), $500 \mathrm{U} / \mathrm{ml}$ collagenase II (Biochrom), 1.5 $\mathrm{U} / \mathrm{ml}$ collagenase $\mathrm{P}$ (Roche, Basel, Switzerland), and $50 \mathrm{U} / \mathrm{ml}$ hyaluronidase (Sigma, Taufkirchen, Germany). Following digestion on a magnetic stirrer, the cell suspension was strained through nylon mesh with a pore diameter of $100 \mu \mathrm{m}$ (Becton 
Dickinson, Heidelberg, Germany). The isolated cells were washed twice in Hank's salt solution (Biochrom), centrifuged, and resuspended in RPMI 1640 medium containing 10\% FBS and $200 \mu \mathrm{M}$ L-ascorbic acid 2-phosphate (AsAP; Sigma).

\section{Cell seeding}

Manual and automated cell seeding were compared using a suspension of primary chondrocytes. The concentration of isolated and harvested chondrocytes was adjusted to $0.4 \times 10^{6}$ cells/ml in RPMI 1640 medium. For comparison of automated and manual seeding, $200 \mu \mathrm{l}$ of the cell suspension was placed into each well of a 96-well tissue culture plate either automatically with the $\mathrm{CyBi}^{\mathrm{TM}}$-Disk workstation (CyBio AG, Jena, Germany) or manually using a multichannel pipette ( 8 channels). The number of viable cells in each well was determined photometrically using an MTS proliferation assay following the manufacturer's instructions (Promega, Madison, WI). In brief, $40 \mu \mathrm{l}$ of the MTS solution was added to a 200- $\mu \mathrm{l}$ cell suspension with the $\mathrm{CyBi}^{\mathrm{TM}}$-Disk workstation for both seeding methods. The plates were incubated for another $3 \mathrm{~h}$ in a humidified incubator. Subsequently, $100 \mu \mathrm{l}$ of the suspension was transferred into new microplates also using the $\mathrm{CyBi}^{\mathrm{TM}}$-Disk workstation for both methods. The absorbance was recorded at $492 \mathrm{~nm}$ against a reference wavelength of $620 \mathrm{~nm}$ using an enzyme-linked immunosorbent assay 96-well microplate reader (Anthos Labtec Instruments, Wals, Austria).

\section{Preparation of pellet cultures}

For formation of pellet cultures, $200 \mu \mathrm{l}$ of a cell suspension containing $3 \times 10^{6}$ chondrocytes per milliliter were placed into each well of an uncoated, flat-bottom, 96-well tissue culture plate (Becton Dickinson) using a multichannel pipette. Subsequently, the culture plates were transferred into a humidified incubator to ensure sedimentation of the chondrocytes. The cultures were maintained in RPMI 1640 containing $10 \%$ FBS and $200 \mu \mathrm{M}$ AsAP. On the following 3 days, $100 \mu \mathrm{l}$ of medium was exchanged. During the remaining 10 days of pellet cultures, $200 \mu \mathrm{l}$ was replaced with fresh medium every day. For comparison, the replacement of the medium was performed either automatically with the $\mathrm{CyBi}^{\mathrm{TM}}$-Disk workstation or manually using a multichannel pipette.

\section{Preparation of interactive co-cultures}

After 2 weeks of pellet culture, $200 \mu \mathrm{l}$ of culture medium was exchanged with $200 \mu \mathrm{l}$ of cell suspension containing $0.3 \times 10^{6}$ cells/ml of 1 of 2 human cell lines: 1) SV40T antigen-transformed SFs derived from an RA patient (HSE cell line) or 2) SV40T antigen-transformed SFs derived from a healthy donor (K4IM cell line). ${ }^{14}$ Subsequently, the microplates were transferred into a humidified incubator to ensure sedimentation of the SFs on top of the chondrocyte pellets and formation of interactive co-cultures.
The co-cultures were maintained in complete RPMI 1640 medium without AsAP. On the first day of co-cultivation, $150 \mu \mathrm{l}$ was replaced with fresh medium. During the remaining 13 days, $200 \mu \mathrm{l}$ was exchanged every day. Again, the media exchange was performed either automatically with the $\mathrm{CyBi}^{\mathrm{TM}}$-Disk workstation or manually using a multichannel pipette. Every day after media exchange, the microplates were scanned in color with a resolution of 600 dpi for optical analysis. For histological, immunohistochemical, and gene expression analysis, pellet samples were taken with tweezers.

\section{Histological and immunohistochemical analysis}

For investigation of cartilage matrix molecules, pellet samples were cut in half and embedded vertically in Tissue Tek $^{\circledR}$ O.C.T. Compound (Sakura, Zoeterwoude, Netherlands). Cryosections that were $6 \mu \mathrm{m}$ thick were mounted on aminoalkylsilane-coated slides. Proteoglycans were stained with Alcian blue 8GX (Roth, Karlsruhe, Germany) at pH 2.5 and counterstained with nuclear fast red (DAKO, Hamburg, Germany). For immunohistochemistry of collagen type I and II, the cryosections were incubated with polyclonal antihuman collagen type I and II antibodies (Acris, Hiddenhausen, Germany). Subsequently, antibody staining was detected using the EnVision ${ }^{\mathrm{TM}}$ System, Peroxidase (AEC) Mouse Kit (DAKO) according to the manufacturer's protocol. For distinguishing between porcine chondrocytes and human SFs, the co-cultures were immunohistochemically analyzed for human leukocyte antigen (HLA) ABC class I antigen presentation on the human SF cell lines. Therefore, cryosections of co-cultures were incubated with monoclonal mouse antihuman HLA-ABC antibody (Acris). Subsequently, antibody staining was detected using the EnVision ${ }^{\mathrm{TM}}$ HRP Mouse Kit (DAKO) according to the manufacturer's protocol. Finally, the sections were counterstained with hematoxylin (DAKO). Negative controls were similarly stained by replacing the primary antibody with rabbit immunoglobulin $\mathrm{G}(\mathrm{IgG})$.

\section{Gene expression analysis}

Total RNA from porcine pellet cultures was isolated as described previously. ${ }^{16}$ Five micrograms of total RNA was transcribed reversely after annealing of $0.5 \mu \mathrm{g}$ oligo(dT) primer (Invitrogen, Karlsruhe, Germany) and $200 \mathrm{U}^{\text {SuperScript }}{ }^{\mathrm{TM}}$ reverse transcriptase in $10 \mu \mathrm{l} .{ }^{17}$ The relative expression level of glyceraldehyde-3-phosphate dehydrogenase (GAPDH) was used to normalize the gene expression in each sample. Real-time PCR using the iCycler PCR System (BioRad, München, Germany) was performed with $2 \mu \mathrm{l}$ of the single-stranded complementary DNA sample using the SYBR Green PCR Core Kit (Applied Biosystems, Darmstadt, Germany). Relative quantification of cartilage marker gene expression (Table 1) was performed as described previously ${ }^{18}$ and is given as a percentage of the expression of the GAPDH product. In addition, the relative expression 
Table 1. Oligonucleotide Sequences of Chondrogenic Marker Genes

\begin{tabular}{clc}
\hline \hline Gene & \multicolumn{1}{c}{ Oligonucleotides $\left(5^{\prime} \rightarrow 3^{\prime}\right)$} & $\begin{array}{c}\text { Product } \\
\text { Size, } b p\end{array}$ \\
\hline Aggrecan & CCA GAA TCT AGC AGG GAG TCA TC & 118 \\
& AGG CAG AGG TGG CTT CAG TC & \\
Collagen & CGA TGG CTG CAC GAG TCA CAC & 180 \\
type I & CAG GTT GGG ATG GAG GGA GTT TAC & \\
Collagen & CCG GGC AGA GGG CAA TAG CAG GTT & 128 \\
type II & CAA TGA TGG GGA GGC GTG AG & \\
COMP & GGG TGG CCG CCT GGG GGT CTT & 116 \\
GAPDH & CTT GCC GCA GCT GAT GGG TCT C & \\
& AGG GGC TCT CCA GAA CAT CAT & 117 \\
\hline
\end{tabular}

bp, base pairs; COMP, cartilage oligomeric matrix protein; GAPDH, glyceraldehyde-3-phosphate dehydrogenase.

was related to the relative expression of samples taken at day 1 of the pellet culture.

\section{RESULTS}

\section{Seeding of chondrocytes}

For comparison of automated and manual cell seeding, a chondrocyte suspension was placed in 96-well microplate tissue culture plates using both methods. The number of viable cells per well was determined using the MTS assay (Fig. 2). Automated seeding led to a cell concentration of $0.055 \times 10^{6} \pm 0.0041 \times 10^{6}$ cells/well (coefficient of variation $[\mathrm{CV}]=7.5 \%$ ), which relates to $68.8 \%$ of the expected cell concentration. Manual seeding resulted in a cell concentration of $0.062 \times 10^{6} \pm 0.0044 \times 10^{6}$ cells/well $(\mathrm{CV}=7.1 \%)$. This equals $77.5 \%$ of the expected cell concentration. Therefore, compared with manual seeding, automatic cell seeding in 96-well microplates resulted in a slightly lower mean cell number and a higher CV and thus is less consistent (Table 2).

The time needed for cell seeding was measured for both seeding methods. Cell seeding using the $\mathrm{CyBi}^{\mathrm{TM}}$-Disk workstation took 2 min $32 \mathrm{~s}$ per plate, whereas manual seeding using the multichannel pipette took approximately 3 min $30 \mathrm{~s}$ per plate. Thus, automated seeding saved $28 \%$ of the handling time. In addition, the manual cell-seeding process comprised a higher intensity of labor because of the permanent handling, whereas automated cell seeding required only setting up the $\mathrm{CyBi}^{\mathrm{TM}}$-Disk workstation with the 96-well microplates.

\section{Cultivation of chondrocytes}

Optical analysis. Optical scanning revealed that $18 \%$ of the manually treated cultures detached from the bottom of the well and could not be used for co-cultivation with synovial fibroblasts (Fig. 3A). In comparison, automatically cultivated pellets did not detach from the wells (Fig. 3B). Furthermore, automation of media exchange allowed a flow of $2.3 \mu \mathrm{l} / \mathrm{s}$, whereas manual handling could be performed with a minimum flow of only $10 \mu \mathrm{l} / \mathrm{s}$. Manual pipetting was also nonuniform because of differences between individuals.

Histological and immunohistochemical examination of the pellet cultures. Cryosections of chondrocyte pellets were stained with Alcian blue for proteoglycans and with specific antibodies for collagen type I and II. Figure 4 presents the staining of proteoglycans, collagen type I, and collagen type II after 14 days of manual and automated cultivation in comparison. Both cultivation methods led to a strong secretion of proteoglycans (Fig. 4A, E) and the cartilage-specific collagen type II (Fig. 4B, F), indicating the formation of cartilage-like ECM. Nevertheless, the dedifferentiation marker collagen type I (Fig. 4C, G) was also evident although to a lesser extent. However, manual media exchange led to a stronger collagen type I expression in the pellet margins. The negative IgG controls revealed no staining of the pellets (Fig. 4D, H). For formation of interactive co-cultures, the 14-day-old pellet cultures were coated with the human HSE (Fig. 5A-C) and K4IM (Fig 5D-F) cell lines by sedimentation. The cartilage ECM was detected by Alcian blue staining for proteoglycans, whereas the cell nuclei of chondrocytes and SFs were counterstained with nuclear fast red (Fig. 5A, D). The presence of human SFs was verified by staining of HLA-ABC, which is specific for human nucleated cells (Fig. 5B, E). Both cell lines showed adherence to the chondrocyte pellets. Normal donor-derived K4IM cells formed a smooth layer covering the pellets, whereas HSE cells derived from RA patients showed rather thick and irregular multilayers and seemed to invade the cartilage pellets in some areas. The corresponding IgG-negative controls remained unstained (Fig. 5C, F).

Real-time PCR. To analyze the differentiation level of the chondrocyte pellet cultures during automated and manual cultivation, the gene expression of cartilage matrix proteins was determined by performing a real-time PCR. After normalization with expression level of the housekeeping gene GAPDH, all values were related to the gene expression level of day 1 . Thus, plotting the gene expression level over the cultivation time shows the development in relation to the start of the culture comparing automated and manual media exchange (Fig. 6). In general, automated and manual cultivation revealed a qualitatively similar regulation of the ECM genes within 14 days of pellet culture. Although collagen type I expression was increased during the cultivation (Fig. 6A), the expression of the main cartilage marker collagen type II (Fig. 6B) showed an increase only during the first 3 days of cultivation, followed by downregulation until day 10 . Although downregulation continued in manually treated cultures, automatically handled cultures revealed upregulation of collagen type II expression from day 10 to day 14 , finally leading to a 3 -fold higher expression 


\section{Ibold et al.}
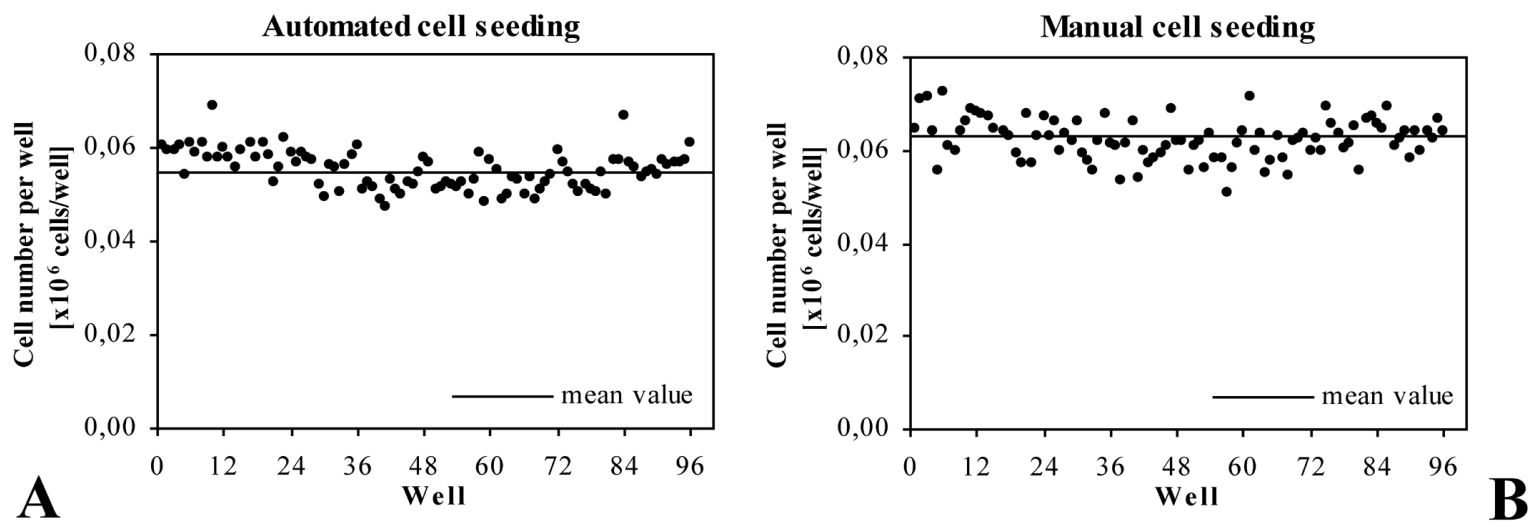

FIG. 2. Comparison of automated (A) and manual (B) seeding of chondrocytes. After seeding with both methods, the number of viable chondrocyte cells number was determined in every well of a 96-well microplate using the MTS assay.

Table 2. Summary of the General Differences between Automated Handling and Manual Handling of the 3-Dimensional Pannus Model in a 96-Well Format

\begin{tabular}{|c|c|c|c|}
\hline & Automated Handling & Manual Handling & $\begin{array}{l}\text { Characteristics of Automated Handling } \\
\text { Compared with Manual Handling }\end{array}$ \\
\hline \multicolumn{4}{|l|}{ Cell seeding } \\
\hline \multicolumn{4}{|l|}{ Accuracy } \\
\hline Volume/well & $-1.9 \%^{\mathrm{a}}$ & $+0.4 \%{ }^{\mathrm{a}}$ & $\begin{array}{l}\text { Lower accuracy, optimization of automated } \\
\text { pipetting volume necessary }\end{array}$ \\
\hline Cell number/well & $68.8 \%$ & $77.5 \%$ & \\
\hline \multicolumn{4}{|l|}{ Reproducibility } \\
\hline $\mathrm{SD}$ & $0.0041 \times 10^{6}$ cells/well & $0.0044 \times 10^{6}$ cells/well & Higher reproducibility \\
\hline \multicolumn{4}{|l|}{ Consistency } \\
\hline Coefficient of variation & $7.5 \%$ & $7.1 \%$ & Lower consistency \\
\hline \multicolumn{4}{|l|}{ Handling time } \\
\hline Pipetting time/plate & $2 \min 32 s$ & $3 \min 30 s$ & $28 \%$ time saving \\
\hline \multicolumn{4}{|l|}{ Cultivation } \\
\hline \multicolumn{4}{|l|}{ Pellet ECM } \\
\hline Cell distribution and morphology & $\begin{array}{l}\text { Homogenous distribution } \\
\text { of round chondrocytes }\end{array}$ & $\begin{array}{l}\text { Homogenous distribution } \\
\text { of round chondrocytes }\end{array}$ & No significant differences \\
\hline ECM composition & $\begin{array}{l}\text { Homogenous, containing } \\
\text { collagen type I and II } \\
\text { and proteoglycans }\end{array}$ & $\begin{array}{l}\text { Homogenous, containing } \\
\text { collagen type I and II } \\
\text { and proteoglycans }\end{array}$ & \\
\hline \multicolumn{4}{|l|}{ Differentiation level } \\
\hline Cartilage marker genes & $\begin{array}{l}\text { Collagen type I and II, } \\
\text { aggrecan, COMP }\end{array}$ & $\begin{array}{l}\text { Collagen type I and II, } \\
\text { aggrecan, COMP }\end{array}$ & $\begin{array}{l}\text { Except for COMP, } 2 \text { to } 3 \text { times higher } \\
\text { expression level after } 14 \text { days }\end{array}$ \\
\hline \multicolumn{4}{|l|}{ Shear forces } \\
\hline Pipetting time/well & $1 \min 27 \mathrm{~s}$ & $20 \mathrm{~s}$ & Reduction of shear forces and failure rate \\
\hline Flow velocity & $2.3 \mu 1 / \mathrm{s}$ & $10 \mu \mathrm{l} / \mathrm{s}$ & \\
\hline Failure rate & $0 \%$ & $18 \%$ & \\
\hline \multicolumn{4}{|l|}{ Handling time } \\
\hline Pipetting time/plate & $7 \min 40 s$ & $9 \min$ & $15 \%$ time saving \\
\hline
\end{tabular}

a. Data not shown.

level compared with manual handling. Although manual cultivation kept aggrecan expression (Fig. 6C) on a higher level within the first week, it was downregulated much more strongly in manually handled cultures in the second week of culture. Thus, after 2 weeks, automated media exchange led to a 2-fold higher expression level of aggrecan. Automated cultivation also led to a downregulation of cartilage oligomeric matrix protein (COMP; Fig. 6D) during 2 weeks of cultivation, whereas manually treated 

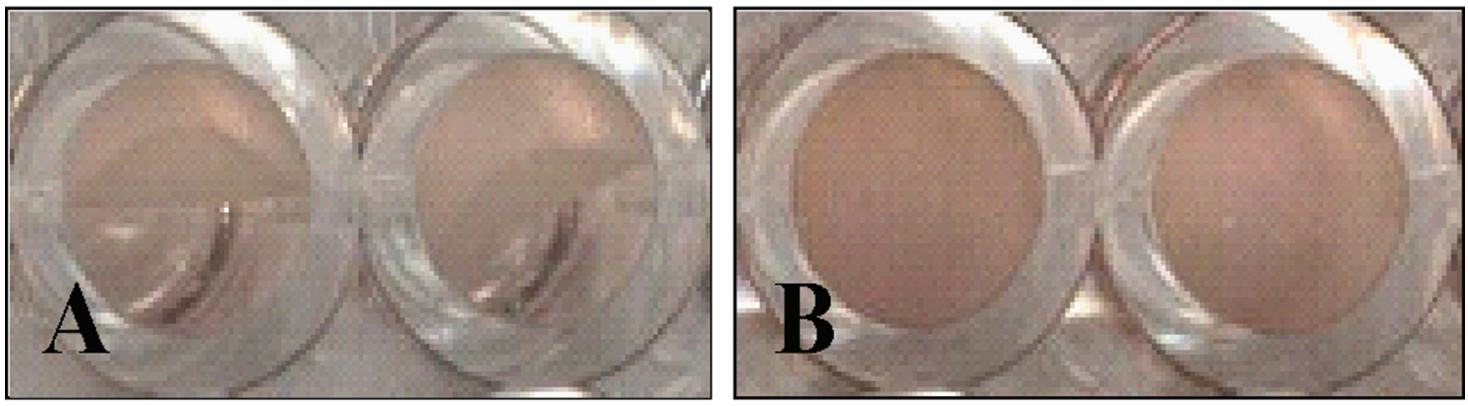

FIG. 3. Optical scans of 9-day-old chondrocyte pellet cultures cultivated either automatically or manually. Although manually handled pellets partly detached from the bottom (A), automatically cultivated chondrocyte pellets did not show any damage or detachment from the well bottom (B).
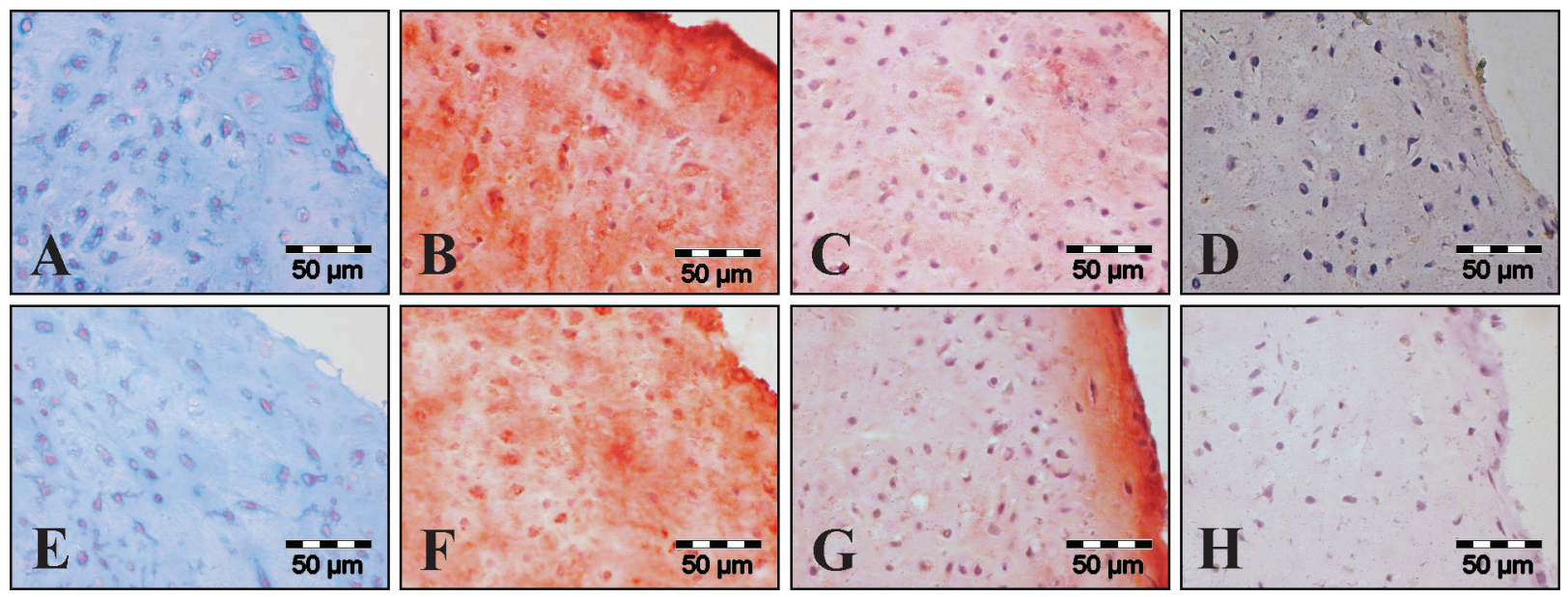

FIG. 4. Histological and immunohistochemical staining of 14-day-old chondrocyte pellet cultures cultivated either automatically (A-D) or manually $(\mathbf{E}, \mathbf{F})$. (A, E) Alcian blue staining of proteoglycans. (B, F) Immunostaining for collagen type II. (C, G) Immunostaining for collagen type I. (D, H) Immunoglobulin G (IgG)-negative control. Both automated and manual cultivation led to strong staining of cartilage-specific proteoglycans and collagen type II and only slight staining of collagen type I. The IgG-negative controls remained unstained.

cultures showed an increase of COMP expression level within the first 3 days. Nevertheless, continued manual cultivation also resulted in downregulation of COMP until day 14. Thus, after 2 weeks of cultivation, both methods resulted in a similar level of COMP expression.

Table 2 summarizes the differences between manual and automated processing of the 3-D pannus cultures regarding cell seeding and cultivation. As important quality criteria for comparison of manual and automated seeding, the accuracy, reproducibility, consistency, and handling time have to be considered. The cultivation process is evaluated by means of analysis of cartilage-specific ECM formation in the pellet cultures, the differentiation level of the chondrocytes, the shear forces, and, again, the handling time.

\section{DISCUSSION}

The 3-D pannus model for RA has already been studied for its functionality, and it has been shown that the process of cartilage destruction can be demonstrated with this in vitro model. ${ }^{6,12,19}$ For this purpose, primary chondrocytes have been cultivated in highdensity cartilage pellets and then co-cultivated with human RASFs. These activated RA-SFs are believed to play a major role in the destruction of joint cartilage during the pathogenesis of RA. ${ }^{2,20}$ For a future application of the 3-D pannus model as an in vitro test system for antirheumatic pharmaceuticals in the drugscreening process, the whole culture process including cell seeding and cultivation was automated.

\section{Automated cell seeding}

Primary chondrocytes were isolated from the cartilage of porcine femur bones. The use of porcine material did not influence the functionality of this test system because the structure of porcine and human articular cartilage is considered to be very similar. ${ }^{21}$ Seeding of the freshly isolated chondrocytes was performed automatically and manually to compare these methods 

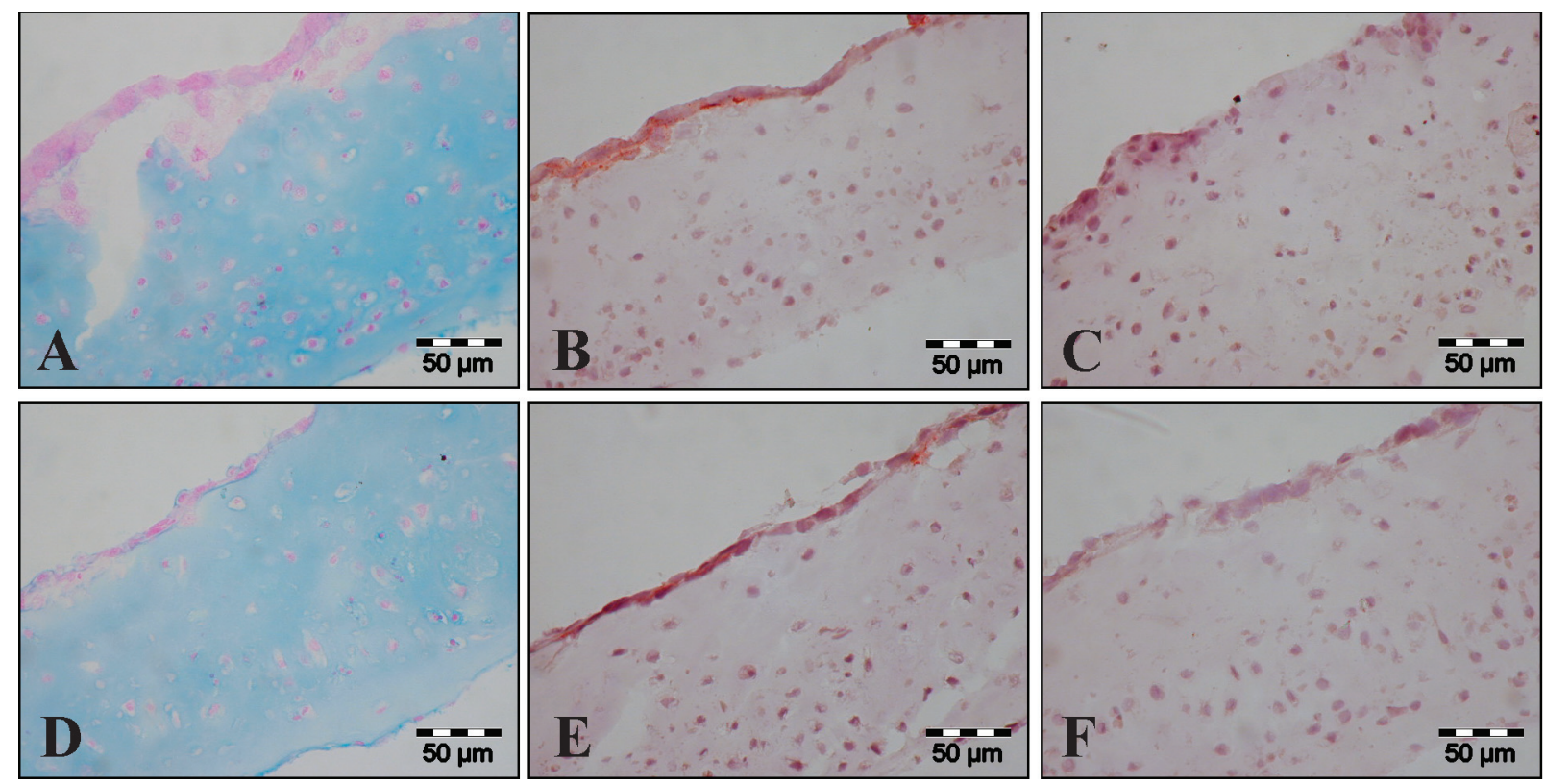

FIG. 5. Histological and immunohistological staining of 28-day-old chondrocyte pellets co-cultivated with either HSE cells (A-C) or K4IM cells (D-F). All cultures were cultivated automatically. (A, D) Alcian blue staining of proteoglycans. (B, E) Immunostaining for human leukocyte antigen (HLA)-ABC surface antigens. (C, E) Immunoglobulin G-negative control. HLA-positive cells, indicated by a red color, could be detected as a layer on top of the chondrocyte pellets, which could already be observed in Alcian blue staining with nuclear fast red counterstaining.

regarding their accuracy, reproducibility, consistency, and required handling time. Although both methods resulted in a seeded cell number per well less than the adjusted cell concentration, manual seeding appeared to be more accurate than automated cell seeding. With 78\%, it showed a cell concentration closer to the seeded stock solution compared to the $\mathrm{CyBi}^{\mathrm{TM}}$-Disk workstation, which reached only $69 \%$ of the adjusted cell concentration. In addition, although the standard deviation of the cell concentration between the single wells of the 96-well microplate was lower after automatic seeding, calculation of the CV revealed that manual handling achieved a slightly better assay consistency. A homogeneous cell distribution over the plate is the basis for a homogeneous ECM formation and is essential to ensure equal test conditions for all pellets in the plate. However, the slightly lower consistency in automatic seeding can still be compensated for by savings in handling time and labor as well as the higher throughput. Moreover, the accurate cellseeding number and volume can still be improved by optimizing the seeding procedure for the $\mathrm{CyBi}^{\mathrm{TM}}$-Disk workstation and by adjusting the pipetting volume.

A further criterion for evaluation of the cell-seeding process is the handling time. To develop an assay for high-throughput screening (HTS), the handling time has to be minimized. This is accomplished by automated seeding, which saves $28 \%$ of the handling time compared with manual handling. In addition, the manual cell-seeding process comprises a higher intensity of labor because of permanent handling, whereas automated cell seeding requires only setting up of the workstation with the 96-well microplates. Manual handling also generates a higher variability because of differences between individuals.

\section{Automated pellet cultivation}

To ensure a high reproducibility for the test system by formation of homogeneous cartilage pellets, it is important to minimize shear forces in the wells during media exchange. In the first 4 days of cultivation, the pellets are very fragile because the cartilage ECM that provides the mechanical strength of the pellets still has to be built up. Higher shear forces especially during this period can lead to an inhomogeneous distribution of the chondrocytes, damage of the pellets, detachment, and even convolving of the pellets. Detached or damaged pellets cannot be used for further co-cultivation because a homogeneous distribution of the synovial component cannot be ensured. Optical scanning of pellet cultures revealed that manual cultivation could damage the fragile pellets due to high shear forces. This led to a failure rate of $18 \%$. The workstation provides a slow and constant media exchange with a flow velocity that was 4 times lower compared with manual handling. The low shear forces generated by this steady pipetting process supported a homogeneous development of the cultures, preventing any damage of the fragile pellets. It also has been shown that mechanical stimulation can alter the chondrocyte metabolism but with controversial results. Several 
A

Collagen type I

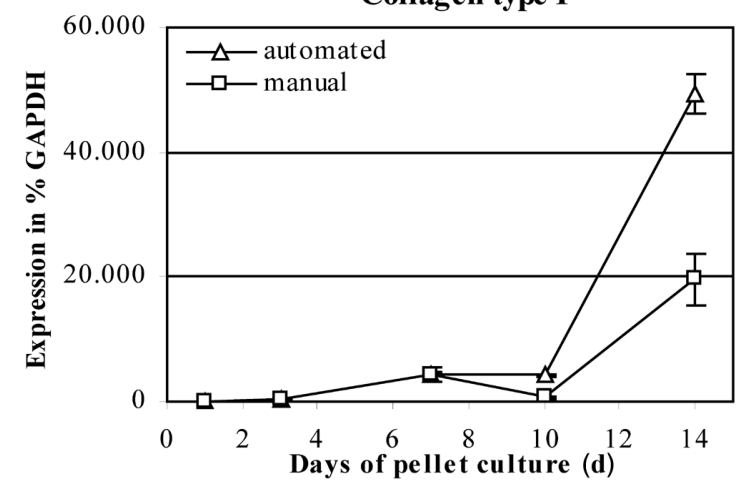

Aggrecan

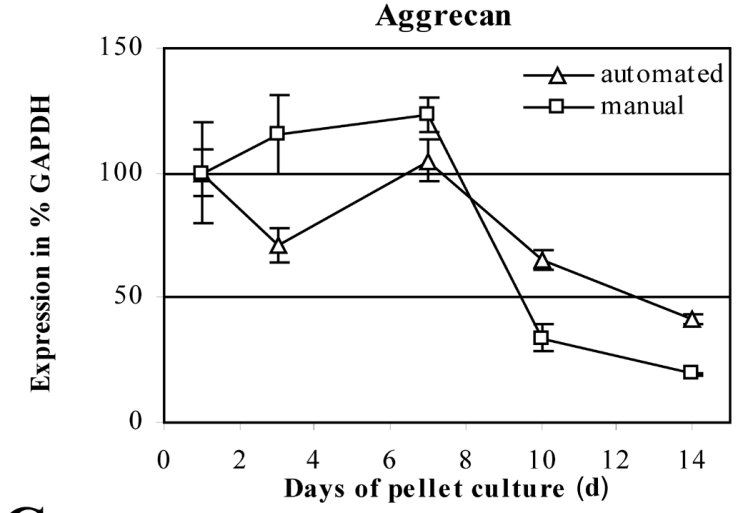

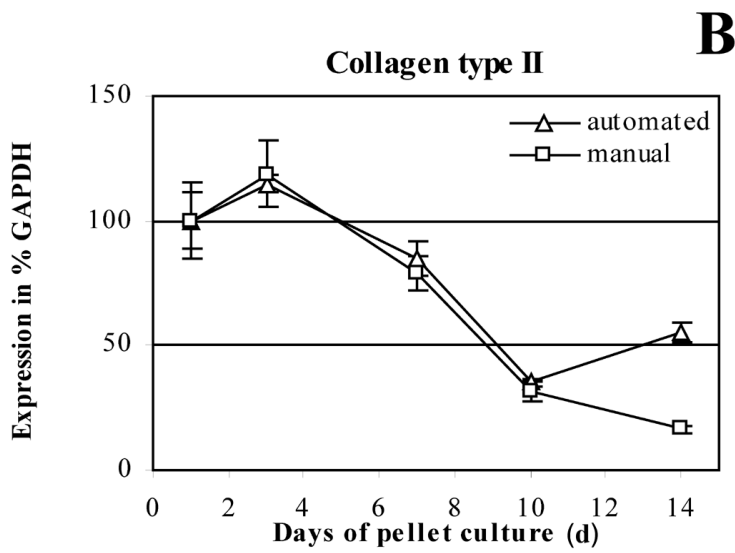

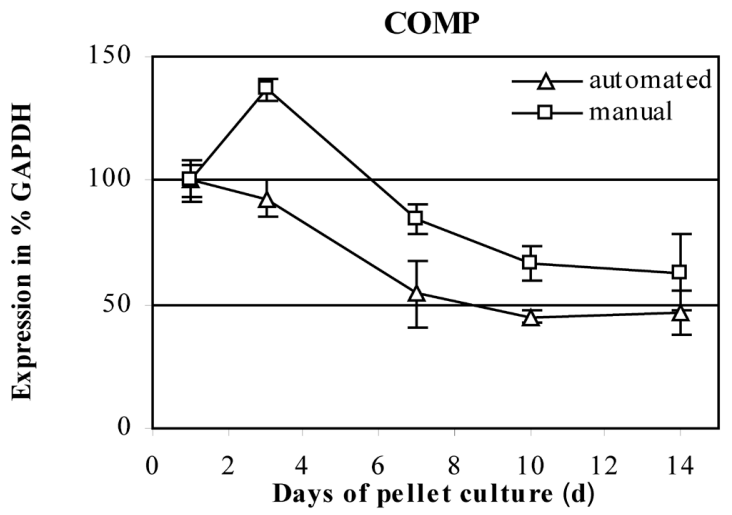

C

FIG. 6. Gene expression analysis of chondrogenic marker genes in porcine high-density pellet cultures cultivated either automatically or manually for 14 days. The mRNA expression of the cartilage matrix genes collagen type I and II, aggrecan, and cartilage oligomeric matrix protein (COMP) was calculated as a percentage of the expression of glyceraldehyde-3-phosphate dehydrogenase (GAPDH) and normalized with the expression of day 1 of the cultivation. Plotting of the gene expression reveals regulation of the genes in comparison to the start of the cultivation.

groups have shown that hydrostatic pressure applied on chondrocytes increased the expression of cartilage ECM proteins such as proteoglycans and collagen type II, whereas shear stress resulted in a decrease of such molecules. ${ }^{22-24}$ Waldman et $\mathrm{al}^{25}$ observed an increase in collagen and proteoglycan synthesis by applying low amplitudes of cyclic shear strain, whereas increasing amplitudes again inhibited synthesis of these proteins. Because the shear forces on automatically treated pellets are smaller than on manually handled pellets, this gives a potential explanation for the slightly better preservation of the hyaline cartilage phenotype seen in the expression analysis.

For further characterization of the pellets, their matrix composition was analyzed by histology and immunohistochemistry. Alcian blue stains acidic proteoglycans in the pellets, which are the second main component of hyaline cartilage, and therefore allows analysis of cell distribution as well as ECM development during the cultivation. Besides the strong expression of proteoglycans, also the spherical round phenotype of the chondrocytes could be preserved in both automatically and manually cultivated pellets. Because the process of chondrocyte dedifferentiation is often accompanied by morphological changes to a fibroblast-like phenotype, ${ }^{4,26,27}$ it can be assumed that the chondrocytes maintained their differentiated state. In addition, immunohistochemical staining for the dedifferentiation marker collagen type I and the specific cartilage marker collagen type II were performed to further analyze the composition of the ECM formed by the primary chondrocytes. Collagens are the main components of articular cartilage, with collagen type II accounting for about $90 \%$ to $95 \%{ }^{28}$ Both automated and manual media exchange led to formation of collagen type II and only a slight secretion of collagen type I. A difference between both handling methods could be seen only in the pellet margins. Whereas automated cultivation revealed a stronger collagen type II staining in the pellet margins, manual media exchange led to a higher content of collagen type I in this area. Thus, it could be concluded that a hyaline-like cartilage ECM was formed by both manual and automated cultivation. 


\section{Ibold et al.}

This cartilage quality conforms to the results found for engineered cartilage from other groups. ${ }^{8,29}$

Moreover, histological analysis of co-cultures with HSE cells showed formation of irregular cell multilayers and partial invasion of the cartilage underneath. This met expectations because HSE cells have a higher proliferation rate than K4IM cells (data not shown) and thus are more likely to form multilayers by extensive growth. Furthermore, it has been reported that RA-SFs are in an activated state, showing features such as stimulation of proliferation, loss of contact inhibition, and oncogene activation. ${ }^{20,30}$ As a negative control, all pellets were also co-cultivated with the K4IM cell line, which was originally derived from a healthy donor. These cells showed no invasive behavior and formed smooth monolayers on top of all cartilage pellets. To ensure that the cell layers on top of the pellets were formed by SFs and not by chondrocytes, immunohistochemistry for the human HLA$\mathrm{ABC}$ surface marker was used. As expected, it could be shown that the human SFs formed the distinct layer on top of the cartilage pellets, whereas the pellets derived from porcine tissue were not stained at all. This has also been shown for pannus cultures in 48-well microplates. ${ }^{12,19}$

The effect of automated cultivation in comparison to manual handling was also investigated by studying the gene expression of important cartilage marker genes using real-time PCR. All values were normalized by the gene expression level of the housekeeping gene GAPDH, which is constitutively expressed. ${ }^{31}$ Both cultivation methods led to a slight but not pronounced upregulation of collagen type II expression in the first 3 days of cultivation, followed by downregulation. The slight increase in collagen type II expression might be related to an initial impulse to compensate for the loss of ECM after digestion. ${ }^{32}$ Loss of collagen type II expression can be explained by beginning dedifferentiation of the chondrocytes. This fact is supported by the kinetics of collagen type I expression, which is strongly upregulated after 3 days of cultivation. However, by day 14, automated media exchange resulted in an expression level of collagen type II that is 3 times higher than the manual media exchange, thus showing a better preservation of the hyaline-specific phenotype. Interestingly, Kolettas and others ${ }^{33}$ showed that dedifferentiation is marked by the loss of ECM markers such as collagen type II rather than by additional expression of nonspecific molecules such as collagen type I. Besides the collagens, one of the most important proteoglycans, aggrecan, was analyzed as well. Automated media exchange resulted in an expression after 2 weeks that was 2 times higher than with manual cultivation. The expression of COMP, one of the major noncollagenous proteins in cartilage, revealed no significant difference between automated and manual handling after 2 weeks of cultivation. Thus, a comparison of the differentiation level in automatically and manually cultivated pellets revealed that with both methods, the hyaline-specific cartilage markers collagen type II, aggrecan, and COMP are expressed but downregulated during the 2 weeks of cultivation, whereas collagen type I was upregulated.
Although the cartilage-specific markers were downregulated, the development of the expression of these markers met expectations because similar results for in vitro engineered cartilage have been reported by others. ${ }^{12,32}$ Nevertheless, automated media exchange led to the same or higher mRNA expression level of all cartilagespecific markers, showing that automated cultivation preserves the chondrocyte differentiation level to a greater extent than manual cultivation does.

In summary, the results show that an automated processing of the 3-D pannus model not only preserves the functionality of the test system but also slightly improves the cartilage quality of the pellet culture. In addition, lowering the shear forces and flow velocities avoids damaging the fragile pellets and ensures constant and reproducible test conditions during the cultivation. Moreover, handling time and labor intensity are strongly reduced, which enables a higher testing frequency. Thus, automated processing offers the possibility to apply the 3-D pannus model as a high-throughput in vitro test system for antirheumatic substances as a transitional step between 2-D cell-based HTS assays and clinical studies.

\section{ACKNOWLEDGMENTS}

This study was supported by grant $0313604 \mathrm{~A} / \mathrm{B}$ from the Bundesministerium für Bildung und Forschung. We are also very grateful to the CyBio AG (Jena, Germany) for providing us with the $\mathrm{CyBi}^{\mathrm{TM}}$-Disk workstation during this study.

\section{REFERENCES}

1. Doan T, Massarotti E: Rheumatoid arthritis: an overview of new and emerging therapies. J Clin Pharmacol 2005;45:751-762.

2. Meinecke I, Rutkauskaite E, Gay S, Pap T: The role of synovial fibroblasts in mediating joint destruction in rheumatoid arthritis. Curr Pharm Des 2005;11:563-568.

3. Roskelley CD, Srebrow A, Bissell MJ: A hierarchy of ECM-mediated signaling regulates tissue-specific gene expression. Curr Opin Cell Biol 1995;7:736-747.

4. Benya PD, Shaffer JD: Dedifferentiated chondrocytes reexpress the differentiated collagen phenotype when cultured in agarose gels. Cell 1982; 30:215-224.

5. Kunz-Schughart LA, Freyer JP, Hofstaedter F, Ebner R: The use of 3-D cultures for high-throughput screening: the multicellular spheroid model. J Biomol Screen 2004;9:273-285.

6. Schultz O, Keyszer G, Zacher J, Sittinger M, Burmester GR: Development of in vitro model systems for destructive joint diseases: novel strategies for establishing inflammatory pannus. Arthritis Rheum 1997;40:1420-1428.

7. Fedewa MM, Oegema TR Jr, Schwartz MH, MacLeod A, Lewis JL: Chondrocytes in culture produce a mechanically functional tissue. $J$ Orthop Res 1998;16:227-236.

8. Zhang Z, McCaffery JM, Spencer RGS, Francomano CA: Hyaline cartilage engineered by chondrocytes in pellet culture: histological, immunohistochemical and ultrastructual analysis in comparison with cartilage explants. J Anat 2004;205:229-237. 
9. Ballok RT, Heydemann A, Wakefield LM, Flanders KC, Roberts AB, Sporn MB: TGF-beta 1 prevents hypertrophy of epiphyseal chondrocytes: regulation of gene expression for cartilage matrix proteins and metalloproteases. Dev Biol 1993;158:411-429.

10. Alini M, Carey D, Hirata S, Grynpas MD, Pidoux I, Poole AR: Cellular and matrix changes before and at the time of calcification in the growth plate studied in vitro: arrest of type $\mathrm{X}$ collagen synthesis and net loss of collagen when calcification is initiated. J Bone Miner Res 1994;9:1077-1087.

11. Jikko A, Murakami H, Takano Y, Iwamoto M, Kato Y: Characterization of the mineralization process in cultures of rabbit growth plate chondrocytes. Dev Biol 1993;156:372-380.

12. Lübke C, Ringe J, Krenn V, Fernahl G, Pelz S, Kreusch-Brinker R, et al: Growth characterization of neo porcine cartilage pellets and their use in an interactive culture model. Osteoarthritis Cartilage 2005;13:478-487.

13. Xu C, Oyajobi BO, Frazer A, Kozaci LD, Russell RG, Hollander AP: Effects of growth factors and interleukin-1 alpha on proteoglycan and type II collagen turnover in bovine nasal and articular chondrocyte pellet cultures. Endocrinology 1996;137:3557-3565.

14. Aicher WK, Dinkel A, Grimbacher B, Haas C, Seydlitz-Kurzbach EV, Peter HH, et al: Serum response elements activate and cAMP responsive elements inhibit expression of transcription factor Egr-1 in synovial fibroblasts of rheumatoid arthritis patients. Int Immunol 1999;11:47-61.

15. Häupl T, Yahyawi M, Lübke C, Ringe J, Rohrlach T, Burmester GR, et al: Gene expression profiling of rheumatoid arthritis synovial cells treated with anti-rheumatic drugs. J Biomol Screen. 2007;12:328-340.

16. Chomczynski P: A reagent for the single-step simultaneous isolation of RNA, DNA and proteins from cell and tissue samples. Biotechniques 1993;15:532-537.

17. Gubler U, Hoffman BJ: A simple and very efficient method for generating cDNA libraries. Gene 1983;25:263-269.

18. ABI Prism 7700. ABI Prism 7700 Sequence Detection System User Bulletin \#2. Darmstadt, Germany: Applied Biosystems, 1997.

19. Smolian H, Thiele S, Kolkenbrock H, Zacher J, Aicher W, Schultz O, et al: Establishment of an in vitro model for rheumatoid arthritis as test system for therapeutical substances. ALTEX 2001;18:265-280.

20. Pap T, Gay RE, Muller-Ladner U, Gay S: Fibroblast biology: role of synovial fibroblasts in the pathogenesis of rheumatoid arthritis. Arthritis Res 2000;2:361-367

21. Frye CA, Yocum DE, Tuan R, Suyana E, Seftor EA, Seftor RE, et al: An in vitro model for studying mechanisms underlying synoviocyte-mediated cartilage invasion in rheumatoid arthritis. Pathol Oncol Res 1996;2:157-166.

22. Lee MS, Trindade MC, Ikenoue T, Schurman DJ, Goodman SB, Smith RL: Effects of shear stress on nitric oxide and matrix protein gene expression in human osteoarthritic chondrocytes in vitro. J Orthop Res 2002;20: $556-561$.
23. Smith RL, Carter DR, Schurman DJ: Pressure and shear differentially alter human articular chondrocyte metabolism: a review. Clin Orthop Relat Res 2004;427(Suppl):S89-S95.

24. Elder SH, Sanders SW, McCulley WR, Marr ML, Shim JW, Hasty KA: Chondrocyte response to cyclic hydrostatic pressure in alginate versus pellet culture. J Orthop Res 2006;24:1-8.

25. Waldman SD, Spiteri CG, Grynpas MD, Pilliar RM, Kandel RA: Longterm intermittent shear deformation improves the quality of cartilaginous tissue formed in vitro. J Orthop Res 2003;21:590-596.

26. von der Mark K, Gauss V, von der Mark H, Muller P: Relationship between cell shape and type of collagen synthesised as chondrocytes lose their cartilage phenotype in culture. Nature 1977;267:531-532.

27. Schnabel M, Marlovits S, Eckhoff G, Fichtel I, Gotzen L, Vécsei V, et al: Dedifferentiation-associated changes in morphology and gene expression in primary human articular chondrocytes in cell culture. Osteoarthritis Cartilage 2002;10:62-70.

28. Temenoff JS, Mikos AG: Review: tissue engineering for regeneration of articular cartilage. Biomaterials 2000;21:431-440.

29. Tare RS, Howard D, Pound JC, Roach HI, Oreffo ROC: Tissue engineering strategies for cartilage generation-micromass and three dimensional cultures using human chondrocytes and a continuous cell line. Biochem Biophys Res Commun 2005;333:609-621.

30. Davis LS: A question of transformation: the synovial fibroblast in rheumatoid arthritis. Am J Pathol 2003;162:1399-1402.

31. Winer J, Jung CK, Shackel I, Williams PM: Development and validation of real-time quantitative reverse transcriptase-polymerase chain reaction for monitoring gene expression in cardiac myocytes in vitro. Anal Biochem 1999;270:41-49.

32. Kaps C, Fuchs S, Endres M, Vetterlein S, Krenn V, Perka C, et al: Molecular characterization of tissue-engineered articular chondrocyte transplants based on resorbable polymer fleece. Orthopade 2004;33:76-85.

33. Kolettas E, Buluwela L, Bayliss MT, Muir HI: Expression of cartilagespecific molecules is retained on long-term culture of human articular chondrocytes. J Cell Sci 1995;108:1991-1999.

Address correspondence to: Jochen Ringe

Tissue Engineering Laboratory and BCRT Department of Rheumatology and Clinical Immunology Charité-Universitätsmedizin Berlin Tucholskystr. 2 10117 Berlin, Germany

E-mail: jochen.ringe@charite.de 